\title{
Determinants for the transition from work into retirement in Europe
}

\author{
Monika Riedel ${ }^{1}$, Helmut Hofer ${ }^{1 *}$ and Birgit Wögerbauer ${ }^{1,2}$
}

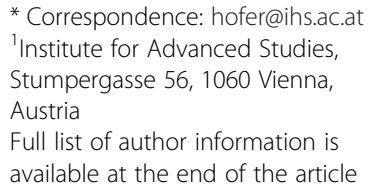

* Correspondence: hofer@ihs.ac.at ${ }^{1}$ Institute for Advanced Studies, Stumpergasse 56, 1060 Vienna, Austria

Full list of author information is available at the end of the article

\begin{abstract}
We use the labor force ad hoc module "transition from work into retirement" to analyze the link between individual as well as pension system characteristics and planned retirement age within the European Union. We find that timing of retirement is correlated between partners who are, already at the stage of retirement planning. In richer countries of the EU15, standard retirement age seems to have a larger effect on planned retirement age than in poorer EU15 states or in new EU member states. The effect of pension wealth is largest in the new EU member states and smallest in rich EU15 countries. Furthermore, industry and occupation effects differ by country type.

JEL codes: $\mathrm{C} 21 ; \mathrm{J} 22 ; \mathrm{J} 26$

Keywords: Planned retirement; Joint retirement decision; Pension system characteristics; Country differences; Europe
\end{abstract}

\section{Introduction}

Increasing longevity is threatening the sustainability of pension systems in many industrialized countries (e.g., Gruber and Wise 1998). In many European countries, the old-age dependency ratio as well as the average duration during which people receive pension benefits has been increasing dramatically over the last years (European Commission 2011). This process is forecasted to continue during the coming decades, thus further adding to the fiscal strain on national pension systems. Across the European Union (EU), the population aged 65 and above will almost double up until the year 2060, while the population aged 15-64 is estimated to drop by $14 \%$ during the same period. For the 27 member states of the EU, public expenditure on pensions have been projected to amount to $12.8 \%$ of the gross domestic product (GDP) by 2060 (European Commission 2012).

Retaining long-run financial sustainability will force several countries to reduce the generosity of their pension systems and to increase the general awareness of pension risks and individual responsibility. Many European countries have already increased the statutory retirement age or are in the process of doing so by changing regulations affecting the effective retirement age. One avenue along this path is to allow the individual worker more flexibility in choosing the retirement age, in order to better account for individual heterogeneity in preferences and productivity. Financial rewards for postponing retirement or creating more options for gradual retirement may be

(c) 2015 Riedel et al.; licensee Springer. This is an Open Access article distributed under the terms of the Creative Commons Attribution License (http://creativecommons.org/licenses/by/4.0), which permits unrestricted use, distribution, and reproduction in any medium, provided the original work is properly credited. 
measures for achieving this goal. In order to establish which policy reforms are apt to reduce the financial pressure on pension systems, it is highly important to understand better which forces or circumstances make workers wish to retire later.

We use the ad hoc module "transitions from work into retirement" for the year 2006 (AHM2006) of the European Labour Force Survey (EU LFS) to analyze the relationship between individual characteristics and the planned retirement age. The ad hoc module is one of the few datasets which satisfies two conditions: First, it includes all member states of the EU, and second, it contains individual-level information relevant for the transition from work to retirement. A special feature of this dataset is that it allows for the investigation of the planned - as opposed to the observed - timing of retirement of still active workers, a question which is increasingly taken into consideration in the literature (see e.g. de Grip et al. 2013; Vonkova and van Soest 2014). Furthermore, the dataset allows us to identify spouses and their retirement plans (if not yet retired), thus enabling us to analyze whether spouses' retirement plans are correlated. As social and economic differences in national backgrounds can be assumed to influence individual retirement decisions, we estimate separate models for three groups of European countries: new members in the EU, richer members of the EU15 (countries with GDP per capita above the EU15 average), and poorer members of the EU15 (countries with GDP per capita below the EU15 average).

This paper contributes to the literature in three ways. First, we analyze how the current transition of the "quality of work" relates to retirement timing. It has been argued that type of work that is based upon empathy and intellectual capacities of workers will increase, while purely physical work will lose importance (Fischer-Kowalski et al. 2012). We operationalize these aspects by using information on occupations and industries. Second, we consider retirement as a decision concerning couples. We investigate the influence of the partner's planned retirement age on own plans for retirement timing. Third, we investigate differences in these effects between country groups in Europe.

Our empirical analysis suggests that intellectual workers and blue-collar workers plan to retire later than other workers, with the effect for intellectual workers being stronger than that for blue collar workers. We find that timing of retirement is positively correlated between partners already at the stage of retirement planning. Moreover, characteristics of the pension system (statutory pension age, generosity of the system) influence the planned age of retirement. The strength of the effects regarding the pension system and the transition of work characteristics indeed differs between country groups.

The paper proceeds as follows. Section 2 offers a short review of the literature on the determinants of retirement expectations and planned retirement decisions. Section 3 describes the data set and outlines our empirical strategy. Section 4 presents our empirical results. The final section discusses our results.

\section{Literature review}

The life-cycle hypothesis predicts that individuals plan on their consumption and savings behavior to maximize utility over their lifetime. According to this theoretical model, the optimal timing for retirement is determined by discount rates and individual risk attitudes (Clark et al. 2012). In reality, however, many factors influence retirement planning. While a large body of empirical literature has been devoted to the ex-post 
analysis of the determinants of retirement, less research is available on retirement planning and the formation of retirement expectations.

A broad body of literature analyses the impact of health on retirement plans. Empirical studies have shown that poor health is associated with early exit from the labor market (e.g., Alavinia and Burdorf 2008; Jones et al. 2010; van den Berg et al. 2010). Furthermore, mental health (problems) has become increasingly important in the retirement discussion (e.g., Alavinia and Burdorf 2008; Karpansalo et al. 2004). A poor health status can be caused either by sudden health shocks, or it can evolve gradually over time. For this reason, an individual's health status is not only important for actual retirement timing, but it also plays an important role for retirement planning. Dwyer and Mitchell (1999), for example, have demonstrated that people who classify themselves as having a poor health status plan to retire on average 1 to 2 years earlier. This finding is in line with McGarry (2004), who also concludes that poor self-reported health has significant effects on retirement planning. Furthermore, McGarry (2004) suggests that changes in health affect retirement expectations stronger than changes in financial variables such as income or wealth. Moreover, it has been found that workers' expectations about their remaining life expectancy affect the retirement planning process. Using Dutch panel data on retirement behavior, van Solinge and Henkens (2009) have revealed that workers with a higher perceived life expectancy plan to retire later (but often fail to realize their plan as firm-side and job pressures prevent them from carrying out their retirement intentions).

Retirement planning is inextricably linked with accumulation of wealth. Without sufficient financial resources to meet one's obligations during retirement, workers simply cannot afford to retire. Thus, wealth not only determines the decision to retire but also affects its timing. This hypothesis has been confirmed by several studies showing that financial factors like wealth and labor income affect the retirement decision (e.g., Dorn and Sousa-Poza 2005; Bütler et al. 2004). Consequently, while early retirement is barely affordable to poorer individuals, richer individuals have the option to accumulate sufficient wealth in order to retire early (Dorn and Sousa-Poza 2005). Existing research shows that individuals' retirement decisions often fail to maximize retirement wealth (Brown 2009). Some authors point out that information biases and financial illiteracy play important roles for retirement planning (e.g., Lusardi and Mitchell 2011; Clark et al. 2012). Accordingly, it has been shown for the US that richer individuals are more successful in retirement planning, while individuals with low educational attainment lack financial literacy and are therefore less successful (Lusardi and Mitchell 2011). A positive relationship between successful retirement planning and financial literacy has also been found for different countries (e.g., Bucher-Koenen and Lusardi 2011 for Germany; van Rooij et al. 2011 for the Netherlands; Almenberg and Säve-Söderbergh 2011 for Sweden). Future pension wealth not only depends on personal financial resources such as wages and wealth, but it is also subject to the generosity of national pension schemes. The relevance of wealth and income thus has to be discussed in the framework of national retirement systems.

Alongside wealth, poor working conditions are among the most discussed determinants of (early) retirement. Several authors (e.g., Siegrist et al. 2007; Elovainio et al. 2005; Schnalzenberger et al. 2008) found a significant positive relationship between early retirement and poor working conditions. Especially workers with jobs characterized by little 
skill variety, little autonomy, and poor social relationships have a higher probability of withdrawing early from the labor force, as compared to workers with intrinsically motivating jobs (Beehr et al. 2000). Accordingly, working conditions are important for retirement planning as "a challenging and rewarding work environment" can motivate older employees to remain longer in the labor market (van Dam et al. 2009). Furthermore, psychosocial factors such as job commitment and attitude towards early retirement have been discussed as important factors for retirement planning. Zappalà et al. (2008), for example, have shown that a positive attitude of individuals towards early retirement induces them to plan for an early retirement. Similarly, high commitment to the firm has been found to be negatively correlated with intended retirement (Adams et al. 2002). Parnes and Sommers (1994) suggest that psychological commitment to one's career and a subsequent preference for work are among the most powerful predictors for continued employment during old age.

A growing literature on the effects of family related issues on retirement suggests that retirement planning should be treated as a household decision rather than an individual decision. As an increasing proportion of married and working women are reaching retirement age, it is important to understand couples' transitions into retirement. Several studies have revealed that retirement is a joint decision of husbands and wives, suggesting that couples coordinate their retirement dates (e.g., Hurd 1990; Gustman and Steinmeier 2000; Zweimüller et al. 1996; Blöndal and Scarpetta 1999; Hospido and Zamarro 2014). These findings can be explained by similar preferences or the wish to spend leisure time together. Furthermore, it has been found that men and women act differently in the retirement decision-making process. For example, women have shown to be more influenced by their partners' resources and retirement expectations than vice versa (Pienta and Hayward 2002). This finding has been confirmed by Moen et al. (2005), who also conclude that women tend to adapt themselves more to their spouses' retirement plans. Men, on the other hand, tend to plan their retirement irrespective of their wives retirement expectations.

Existing literature provides a broad body of evidence suggesting that the design of public old-age pension systems makes a substantial contribution to the low labor force participation rates among older workers (e.g., Gruber and Wise 1998; Blöndal and Scarpetta 1999; Fischer and Sousa-Poza 2006). Especially generosity, actuarial fairness and early exit possibilities of pension systems are widely discussed. While the majority of studies focuses on actual retirement dates, less research has been conducted on the effects of characteristics of national pension systems on retirement planning and retirement expectations. Vonkova and van Soest (2014), for example, used stated preference data on hypothetical retirement scenarios and found that financial incentives (e.g., changes in accruals caused by delayed retirement) can have large effects on the preferred retirement age. Coppola and Wilke (2014) analyzed how increases in the statutory retirement age from 65 to 67 during the 2007 German pension reform influenced retirement expectations of individuals. Results show that retirement expectations have adapted, especially by younger cohorts. The lowest adjustment-rate was found for individuals with a low educational background. Based on these findings, Coppola and Wilke (2014) suggest that individuals react as anticipated and adjust their retirement expectations to altered institutional conditions, provided they are well informed about the reform. This finding is backed up by other studies which also conclude that individuals postpone their expected 
date of retirement according to changes in the statutory retirement age (e.g., Bottazzi et al. 2006 for Italy; de Grip et al. 2013 for the Netherlands).

The discussed studies provide evidence that the determinants of planned retirement and retirement expectations are manifold and interrelated. Generally speaking, planning for retirement is a complex and long-lasting process and is often subject to adjustments over time, be it due to changes in national pension regulations, changes in health or altered financial conditions (Montalto et al. 2000). Despite the complexity of this particular topic, however, retirement expectations of individuals are solid and comply with actual retirement outcomes. Accordingly, several empirical studies (e.g., Bernheim 1987; Benitez-Silva and Dwyer 2005; Disney and Tanner 1999) have shown that expected dates of retirement are relatively accurate. Thus, analyzing planned retirement rather than actual retirement can provide important insights into the retirement timing process.

\section{Data and empirical strategy}

\subsection{Description of the data set}

We use the ad hoc module "transition from work into retirement" for the year 2006 (AHM2006) of the EU-LFS as our main data set. Additional variables are taken from the LFS. This data source was selected for two reasons. First, it covers - at least theoretically - all EU-27 member states as well as Norway, which constitutes a considerable advantage over other possible data sources, like the SHARE data set, which is limited to a smaller number of countries. In the delivered file, however, the dataset of Malta was missing, and we did not use the dataset for Norway due to the focus on EU member states. Missing observations on certain characteristics related to pension systems or job classifications forced us to drop all observations from Romania, Slovenia, Slovakia and the United Kingdom. Second, the AHM2006 is specifically focused on our research question, the transition from work into retirement. AHM2006 includes working as well retired respondents, with some items of the questionnaire specifically adjusted to the respective labor market status: Working individuals are asked about planned transitions, while retired individuals are asked about their actual transition into retirement in the past.

The target group of the AHM2006 consisted of all people aged between 50 and 69, whether they either worked or did not work but worked at least up to the age of 50 . Every LFS respondent of this age group in the respective survey period was included in the sample in most of the countries. Seven countries, however, used sub-samples of the core LFS. Participation in the module was voluntary in most participating countries and compulsory in eight countries ${ }^{1}$.

In our study, we investigate the planned retirement age. Therefore we consider only people from the age group 50-54. Using the full sample would result in biased estimates as plans of individuals in higher ages depend on earlier choices or events. With increasing age, working individuals constitute a biased and increasingly diminishing fraction of their peer group due to prior retirement of the other fraction. In the age group $50-54$, more than $90 \%$ are still working, compared to only $27 \%$ in the age group $60-64$.

The LFS and the AHM2006 have no exact information on a person's age. Information on age at the time of the survey is provided in terms of 5-year age bands only: 50-54, 
$55-59,60-64$, and 65-69. Therefore, we cannot calculate the planned period until a person will retire because the actual age is not contained in the data set.

The LFS AHM2006 contains several possibilities to define retirement depending on the concept of retirement used and on the group of individuals. We decided to use the variable PLAGESTP (planned age for stopping all work for pay or profit) to define planned retirement. This question was asked to all respondents aged 50-69 who fulfil one of two definitions: The first is that they report to have worked for pay or profit (including family workers) during the reference week or did not work even though still having a job or business. The second is that they were on layoff or without job or business during the reference week but had worked until the age of 50. PLAGESTP offers several possibilities to state plans for the timing of retirement, but it also offers the possibility to answer: "has already stopped all work for pay or profit". Note that this definition requires having stopped all work as opposed to having stopped just the main job.

PLAGESTP is available as an exact number ranging from 50 to 93. People unwilling or unable to state an exact age were offered broad age groups (before 60, between 60 and 64,65 or older or planning to work as long as possible). For our evaluations, we recoded these items as "57" (for: before 60), "62" (for: between 60 and 64) and "67" (for: 65 or older) in order not to lose these observations for the econometric analysis. We chose these values for recoding in order to get as close to the assumed average of possible plans as possible. Remember that everybody in our sample is in age group 5054 ; therefore, we think that " 57 " is a more realistic assumed average than, e.g., " 55 ", a planned retirement age that close to the actual age might be more specific already. We chose " 67 " rather than an older age for recoding " 65 or older" because observations on people working far longer than age 70 are very scarce ${ }^{2}$.

To define the labor market status for "working", we use the variable WSTATOR of the LFS which refers to the labor market status during the reference week. We allocate the labor market status "working" to individuals who ticked one of the following two possibilities:

1 Did any work for pay or profit during the reference week - one hour or more (including family workers but excluding conscripts on compulsory military or community service).

2 Was not working but had a job or business from which he/she was absent during the reference week (including family workers but excluding conscripts on compulsory military or community service).

This definition fits well with the definition of retirement using PLAGESTP. Both variables draw the line between "work" and "retirement" according to the same logic: paid work for a very low number of hours is defined as work, not retirement. Or put differently, for being defined as "working" it is not necessary that "work" provides the main income; it is sufficient that one is still economically active.

The combination of the AHM2006 with variables included in the core LFS allows us to include a number of job-related characteristics. We use industry and occupation to reflect the transition of the quality of work as discussed in Fischer-Kowalski et al. (2012). Their storyline is that type of work that is based upon empathy and intellectual capacities of workers will gain importance during the next (or possibly already happening) transition, 
while purely physical work will lose importance. We define four industries: agriculture (NACE A, B), manufacturing (NACE C, D, E, F), personal services (NACE M, N, P). Remaining NACE categories form the base category "other services". We define three occupational classes: blue collar (ISCO 6, 7, 8, 9), intellectual workers (ISCO 1, 2), and a base category of other workers including ISCO 3 through 5.

\subsection{Empirical strategy}

We estimate linear regression models for the planned age at exit from the labor market, which is continuous and observable for every person in the sample. The analysis of a duration process typically requires special econometric techniques to care for censoring in the data, i.e., the process may still be on-going. In our case, this is not a problem because all individuals state a planned retirement date. Moreover, normality of the residuals should not be a problem either. For simplicity reasons, we therefore use an OLS regression technique with robust standard errors.

We are interested in factors related to the pension system and in the stability of the influence of these factors across countries. Factors related to the pension system are, however, not available in the AHM2006 or can not be calculated from it; we therefore cannot include pension-specific information on an individual level. In order to include at least some information describing national pension systems, we use macro-level information from the OECD (2011), i.e., standard retirement age and either replacement rate or pension wealth for the median worker. All variables are available separately for men and women, but not for all EU27 countries.

To analyze country effects, we segregate the sample into more homogeneous groups of countries and run separate regressions in each group of countries. It has been shown that macroeconomic conditions also play some role in national levels of voluntary and involuntary early retirement (Dorn and Sousa-Poza 2010). We assume that economic downturns, which are important for involuntary early retirement of older workers, usually hit European countries roughly at the same time and are therefore not a sensible distinguishing feature to form country groups. The relative level of a country's wealth, however, instrumentalized by per capita GDP, is positively related to voluntary early retirement (Dorn and Sousa-Poza 2010) and therefore forms a more sensible base for a country grouping. We defined three groups of countries: new members in the EU (Bulgaria, the Czech Republic, Estonia, Hungary, Latvia, Lithuania, and Poland), richer members of the EU15 (Austria, Belgium, Denmark, Germany, Finland, the Netherlands, Ireland, Luxembourg and Sweden, countries with GDP per capita above the EU15 average), and poorer members of the EU15 (Spain, Greece, France, Italy and Portugal, countries with GDP per capita below EU15 average). Running separate regressions for each country group allows us to compare size and significance of coefficients across country groups.

\section{Empirical results}

\subsection{Descriptives}

The dataset used consists of 58,091 observations, 54\% male and 46\% female. Observations from poorer EU15 member states (MS) are slightly over-represented; observations from new EU MS are somewhat under-represented, see Table 1. 
Table 1 Sample size and planned age of retirement by country group and sex

\begin{tabular}{lcccc}
\hline & \multicolumn{4}{c}{$\begin{array}{l}\text { Planned age at retirement } \\
\text { Mean (standard deviation) }\end{array}$} \\
\cline { 2 - 5 } & All countries & EU15, rich & EU15, poor & New EU MS \\
\hline Men & $63.0(3.62)$ & $62.8(3.32)$ & $63.0(4.04)$ & $63.3(3.27)$ \\
Women & $61.8(3.81)$ & $62.2(3.34)$ & $62.1(3.93)$ & $60.9(4.01)$ \\
N \% & 100.0 & 33.1 & 38.7 & 28.2 \\
N (Men) & 31,514 & 10,465 & 12,900 & 8,149 \\
N (Women) & 26,577 & 8,780 & 9,561 & 8,236 \\
\hline
\end{tabular}

Source: Author's calculation using AHM2006.

Figure 1 shows the distribution of planned age of retirement. Averages of planned age of retirement do not deviate much between country groups. The gender gap within country groups is largest in new EU MS, with female workers on average planning to retire 2.3 years earlier than male workers. In rich EU15 countries, there is only a gender gap of 0.6 years. Plans to retire on average at age 62-63 may seem to be unremarkable at first glance. It therefore seems noteworthy that already a quarter of all individuals in our sample plans to retire after age 65 , which in many countries still serves as the standard retirement age. Seen from a different angle, we find that $60 \%$ of all workers in our sample plan to stop all paid work before they turn 65 (and another 14\% exactly at age 65).

\subsection{Regression results}

\subsubsection{Quality of work and pension system factors}

Tables 2 and 3 show the effects of individual characteristics and elements of the pension system on the planned retirement age. We start with the results on individual characteristics, which we use as control variables. Our estimates corroborate several well-known findings from the literature. Women plan earlier retirement than men, which is in accordance with (still ongoing) sex-specific legislation in several EU countries. Married individuals also plan earlier retirement than single or widowed individuals, an effect which is far stronger for women. Having achieved higher educational levels seems to be related to a longer active work-life, but this effect does not seem to

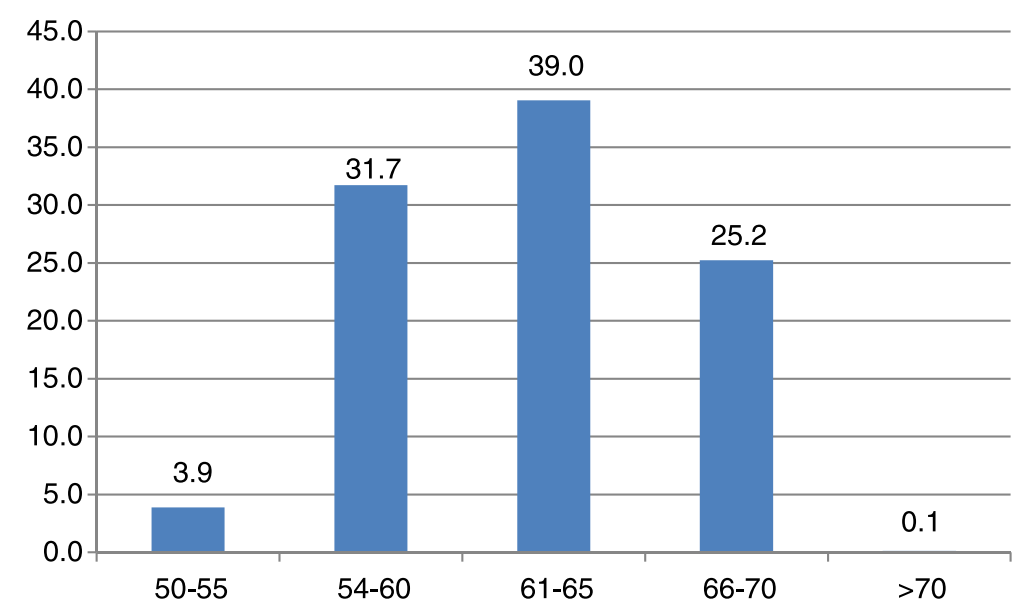

Figure 1 Distribution of planned age at retirement by age brackets, total sample. 
Table 2 Linear regression model estimations for planned retirement age, pension variables: standard retirement age SRA and replacement rate RR

\begin{tabular}{|c|c|c|c|c|c|c|}
\hline & $\begin{array}{l}\text { M1a: total } \\
\text { sample }\end{array}$ & M2a: male & M3a: female & $\begin{array}{l}\text { M4a: EU15, } \\
\text { rich }\end{array}$ & $\begin{array}{l}\text { M5a: EU15, } \\
\text { poor }\end{array}$ & $\begin{array}{l}\text { M6a: new } \\
\text { EU members }\end{array}$ \\
\hline Female & $\begin{array}{l}-0.490^{* * *} \\
(0.0431)\end{array}$ & & & $\begin{array}{l}-0.493^{* * *} \\
(0.0620)\end{array}$ & $\begin{array}{l}0.459^{* * *} \\
(0.0802)\end{array}$ & $\begin{array}{l}-1.523^{* * *} \\
(0.0977)\end{array}$ \\
\hline Married & $\begin{array}{l}-0.460^{* * *} \\
(0.0399)\end{array}$ & $\begin{array}{l}-0.117^{*} \\
(0.0571)\end{array}$ & $\begin{array}{l}-0.726^{* * *} \\
(0.0562)\end{array}$ & $\begin{array}{l}-0.612^{* * *} \\
(0.0540)\end{array}$ & $\begin{array}{l}-0.391^{* * *} \\
(0.0764)\end{array}$ & $\begin{array}{l}-0.351^{* * *} \\
(0.0851)\end{array}$ \\
\hline $\begin{array}{l}\text { Medium } \\
\text { education }\end{array}$ & $\begin{array}{l}-0.0369 \\
(0.0416)\end{array}$ & $\begin{array}{l}0.0596 \\
(0.0544)\end{array}$ & $\begin{array}{l}-0.213^{* *} \\
(0.0653)\end{array}$ & $\begin{array}{l}0.0808 \\
(0.0618)\end{array}$ & $\begin{array}{l}-0.110 \\
(0.0740)\end{array}$ & $\begin{array}{l}0.412^{* * *} \\
(0.0990)\end{array}$ \\
\hline $\begin{array}{l}\text { High } \\
\text { education }\end{array}$ & $\begin{array}{l}0.224^{* * * *} \\
(0.0575)\end{array}$ & $\begin{array}{l}0.0792 \\
(0.0773)\end{array}$ & $\begin{array}{l}0.276^{* *} \\
(0.0881)\end{array}$ & $\begin{array}{l}0.376^{* * *} \\
(0.0782)\end{array}$ & $\begin{array}{l}0.0924 \\
(0.104)\end{array}$ & $\begin{array}{l}1.188^{* * *} \\
(0.168)\end{array}$ \\
\hline Large firm & $\begin{array}{l}-0.407^{* * *} \\
(0.0371)\end{array}$ & $\begin{array}{l}-0.466 \\
(0.0508)\end{array}$ & $\begin{array}{l}-0.318^{* * *} \\
(0.0542)\end{array}$ & $\begin{array}{l}-0.285^{* * *} \\
(0.0504)\end{array}$ & $\begin{array}{l}-0.542^{* * *} \\
(0.0734)\end{array}$ & $\begin{array}{l}-0.242^{* *} \\
(0.0761)\end{array}$ \\
\hline No firmsize & $\begin{array}{l}0.154^{*} \\
(0.0758)\end{array}$ & $\begin{array}{l}0.149 \\
(0.0892)\end{array}$ & $\begin{array}{l}0.182 \\
(0.140)\end{array}$ & $\begin{array}{l}0.563^{* * *} \\
(0.127)\end{array}$ & $\begin{array}{l}-0.187 \\
(0.106)\end{array}$ & $\begin{array}{l}0.00472 \\
(0.175)\end{array}$ \\
\hline Employee & $\begin{array}{l}-1.074^{* * *} \\
(0.0680)\end{array}$ & $\begin{array}{l}-1.032^{* * *} \\
(0.0808)\end{array}$ & $\begin{array}{l}-1.059^{* * *} \\
(0.125)\end{array}$ & $\begin{array}{l}-0.450^{* * * *} \\
(0.110)\end{array}$ & $\begin{array}{l}-1.753^{* * *} \\
(0.104)\end{array}$ & $\begin{array}{l}-0.123 \\
(0.146)\end{array}$ \\
\hline Part-time job & $\begin{array}{l}-0.395^{* * *} \\
(0.0524)\end{array}$ & $\begin{array}{l}-0.0858 \\
(0.111)\end{array}$ & $\begin{array}{l}-0.427^{* * *} \\
(0.0610)\end{array}$ & $\begin{array}{l}-0.268^{* * *} \\
(0.0645)\end{array}$ & $\begin{array}{l}-0.206 \\
(0.115)\end{array}$ & $\begin{array}{l}-0.152 \\
(0.185)\end{array}$ \\
\hline $\begin{array}{l}\text { Working } \\
\text { conditions }\end{array}$ & $\begin{array}{l}-0.00822 \\
(0.0395)\end{array}$ & $\begin{array}{l}-0.0667 \\
(0.0519)\end{array}$ & $\begin{array}{l}0.0791 \\
(0.0609)\end{array}$ & $\begin{array}{l}0.128^{*} \\
(0.0558)\end{array}$ & $\begin{array}{l}-0.0690 \\
(0.0719)\end{array}$ & $\begin{array}{l}-0.0628 \\
(0.0825)\end{array}$ \\
\hline Large city & $\begin{array}{l}0.00212 \\
(0.0410)\end{array}$ & $\begin{array}{l}-0.00802 \\
(0.0552)\end{array}$ & $\begin{array}{l}-0.00281 \\
(0.0612)\end{array}$ & $\begin{array}{l}0.103 \\
(0.0595)\end{array}$ & $\begin{array}{l}-0.0200 \\
(0.0780)\end{array}$ & $\begin{array}{l}-0.156 \\
(0.0849)\end{array}$ \\
\hline Medium city & $\begin{array}{l}-0.242^{* * *} \\
(0.0432)\end{array}$ & $\begin{array}{l}-0.353^{* * *} \\
(0.0573)\end{array}$ & $\begin{array}{l}-0.0895 \\
(0.0657)\end{array}$ & $\begin{array}{l}-0.309^{* * *} \\
(0.0646)\end{array}$ & $\begin{array}{l}-0.137 \\
(0.0806)\end{array}$ & $\begin{array}{l}-0.354^{* * *} \\
(0.0834)\end{array}$ \\
\hline Agriculture & $\begin{array}{l}0.334^{* * *} \\
(0.0826)\end{array}$ & $\begin{array}{l}0.349^{* * *} \\
(0.0987)\end{array}$ & $\begin{array}{l}0.293^{*} \\
(0.149)\end{array}$ & $\begin{array}{l}0.309^{*} \\
(0.142)\end{array}$ & $\begin{array}{l}0.982^{* * *} \\
(0.132)\end{array}$ & $\begin{array}{l}-0.603^{* * *} \\
(0.150)\end{array}$ \\
\hline Manufacturing & $\begin{array}{l}-0.0385 \\
(0.0446)\end{array}$ & $\begin{array}{l}0.0223 \\
(0.0531)\end{array}$ & $\begin{array}{l}-0.215^{* *} \\
(0.0830)\end{array}$ & $\begin{array}{l}0.0252 \\
(0.0642)\end{array}$ & $\begin{array}{l}-0.198^{*} \\
(0.0816)\end{array}$ & $\begin{array}{l}-0.0939 \\
(0.0854)\end{array}$ \\
\hline $\begin{array}{l}\text { Personal } \\
\text { services }\end{array}$ & $\begin{array}{l}0.0907^{*} \\
(0.0456)\end{array}$ & $\begin{array}{l}0.146 \\
(0.0756)\end{array}$ & $\begin{array}{l}0.0142 \\
(0.0584)\end{array}$ & $\begin{array}{l}0.353^{* * *} \\
(0.0610)\end{array}$ & $\begin{array}{l}-0.120 \\
(0.0846)\end{array}$ & $\begin{array}{l}-0.372^{* * *} \\
(0.108)\end{array}$ \\
\hline $\begin{array}{l}\text { Blue collar } \\
\text { worker }\end{array}$ & $\begin{array}{l}0.187^{* * *} \\
(0.0459)\end{array}$ & $\begin{array}{l}0.333^{* * *} \\
(0.0607)\end{array}$ & $\begin{array}{l}0.0442 \\
(0.0740)\end{array}$ & $\begin{array}{l}-0.00734 \\
(0.0656)\end{array}$ & $\begin{array}{l}0.247^{* *} \\
(0.0840)\end{array}$ & $\begin{array}{l}0.0263 \\
(0.0914)\end{array}$ \\
\hline $\begin{array}{l}\text { Intellectual } \\
\text { worker }\end{array}$ & $\begin{array}{l}0.264^{* * *} \\
(0.0501)\end{array}$ & $\begin{array}{l}0.481^{* * *} \\
(0.0676)\end{array}$ & $\begin{array}{l}0.0214 \\
(0.0761)\end{array}$ & $\begin{array}{l}0.0912 \\
(0.0653)\end{array}$ & $\begin{array}{l}0.393^{* * *} \\
(0.0940)\end{array}$ & $\begin{array}{l}0.248 \\
(0.129)\end{array}$ \\
\hline YEARSPNT & $\begin{array}{l}-0.0777^{* * *} \\
(0.00291)\end{array}$ & $\begin{array}{l}-0.109^{* * *} \\
(0.00448)\end{array}$ & $\begin{array}{l}-0.0603^{* * *} \\
(0.00383)\end{array}$ & $\begin{array}{l}-0.0330^{* * *} \\
(0.00394)\end{array}$ & $\begin{array}{l}-0.122^{* * *} \\
(0.00477)\end{array}$ & $\begin{array}{l}-0.0446^{* * *} \\
(0.00988)\end{array}$ \\
\hline SRA & $\begin{array}{l}0.396^{* * *} \\
(0.00856)\end{array}$ & $\begin{array}{l}0.428^{* * *} \\
(0.0150)\end{array}$ & $\begin{array}{l}0.378^{* * *} \\
(0.0106)\end{array}$ & $\begin{array}{l}0.520^{* * *} \\
(0.0218)\end{array}$ & $\begin{array}{l}0.538^{* * *} \\
(0.0164)\end{array}$ & $\begin{array}{l}0.437^{* * *} \\
(0.0247)\end{array}$ \\
\hline $\begin{array}{l}\text { Replacement } \\
\text { rate }\end{array}$ & $\begin{array}{l}-2.033^{* * *} \\
(0.0970)\end{array}$ & $\begin{array}{l}-2.346^{* * *} \\
(0.129)\end{array}$ & $\begin{array}{l}-1.738^{* * *} \\
(0.148)\end{array}$ & $\begin{array}{l}-2.231^{* * *} \\
(0.124)\end{array}$ & $\begin{array}{l}-3.147^{* * *} \\
(0.211)\end{array}$ & $\begin{array}{l}-1.339^{* * *} \\
(0.366)\end{array}$ \\
\hline constant & $\begin{array}{l}2.497^{* * *} \\
(0.558)\end{array}$ & $\begin{array}{l}1.241 \\
(0.976)\end{array}$ & $\begin{array}{l}2.759^{* * * *} \\
(0.673)\end{array}$ & $\begin{array}{l}-7.667^{* * *} \\
(1.419)\end{array}$ & $\begin{array}{l}-4.109^{* * *} \\
(1.039)\end{array}$ & $\begin{array}{l}-1.860 \\
(1.519)\end{array}$ \\
\hline N & 44,558 & 24,493 & 20,065 & 18,905 & 16,719 & 8,934 \\
\hline$R^{2}$ & 0.114 & 0.103 & 0.099 & 0.088 & 0.156 & 0.179 \\
\hline
\end{tabular}

Notes: Standard errors in parentheses. ${ }^{*} p<0.05,{ }^{* *} p<0.01,{ }^{* * *} p<0.001$.

be significant for all Europeans. Women and workers in the more wealthy parts of EU15, but also in new member countries, seem to experience the association between longer working lives and higher education, but male workers and workers in the less wealthy parts of the EU15 do not. In our models, education coefficients are typically significant for higher but not for medium levels of education. 
Table 3 Linear regression model estimations for planned retirement age, pension variables: standard retirement age SRA and pension wealth

\begin{tabular}{|c|c|c|c|c|c|c|}
\hline & $\begin{array}{l}\text { M1b: total } \\
\text { sample }\end{array}$ & M2b: male & M3b: female & $\begin{array}{l}\text { M4b: EU15, } \\
\text { rich }\end{array}$ & $\begin{array}{l}\text { M5b: EU15, } \\
\text { poor }\end{array}$ & $\begin{array}{l}\text { M6b: new } \\
\text { EU members }\end{array}$ \\
\hline Female & $\begin{array}{l}-0.323^{* * *} \\
(0.0434)\end{array}$ & & & $\begin{array}{l}-0.307^{* * *} \\
(0.0616)\end{array}$ & $\begin{array}{l}0.382^{* * *} \\
(0.0797)\end{array}$ & $\begin{array}{l}-1.296^{* * *} \\
(0.118)\end{array}$ \\
\hline Married & $\begin{array}{l}-0.501^{* * *} \\
(0.0400)\end{array}$ & $\begin{array}{l}-0.209^{* * *} \\
(0.0572)\end{array}$ & $\begin{array}{l}-0.737^{* * *} \\
(0.0564)\end{array}$ & $\begin{array}{l}-0.614^{* * *} \\
(0.0541)\end{array}$ & $\begin{array}{l}-0.452^{* * *} \\
(0.0758)\end{array}$ & $\begin{array}{l}-0.357^{* * *} \\
(0.0851)\end{array}$ \\
\hline $\begin{array}{l}\text { Medium } \\
\text { education }\end{array}$ & $\begin{array}{l}-0.0292 \\
(0.0415)\end{array}$ & $\begin{array}{l}0.0759 \\
(0.0543)\end{array}$ & $\begin{array}{l}-0.210^{* *} \\
(0.0654)\end{array}$ & $\begin{array}{l}0.0443 \\
(0.0622)\end{array}$ & $\begin{array}{l}-0.0112 \\
(0.0737)\end{array}$ & $\begin{array}{l}0.410^{* * *} \\
(0.0990)\end{array}$ \\
\hline $\begin{array}{l}\text { High } \\
\text { education }\end{array}$ & $\begin{array}{l}0.235^{* * *} \\
(0.0575)\end{array}$ & $\begin{array}{l}0.149 \\
(0.0775)\end{array}$ & $\begin{array}{l}0.264^{* *} \\
(0.0880)\end{array}$ & $\begin{array}{l}0.331^{* * *} \\
(0.0787)\end{array}$ & $\begin{array}{l}0.168 \\
(0.104)\end{array}$ & $\begin{array}{l}1.172^{* * *} \\
(0.167)\end{array}$ \\
\hline Large firm & $\begin{array}{l}-0.305^{* * *} \\
(0.0373)\end{array}$ & $\begin{array}{l}-0.337^{* * *} \\
(0.0512)\end{array}$ & $\begin{array}{l}-0.259^{* * *} \\
(0.0546)\end{array}$ & $\begin{array}{l}-0.276^{* * *} \\
(0.0505)\end{array}$ & $\begin{array}{l}-0.396^{* * *} \\
(0.0727)\end{array}$ & $\begin{array}{l}-0.239^{* *} \\
(0.0759)\end{array}$ \\
\hline No firmsize & $\begin{array}{l}0.164^{*} \\
(0.0757)\end{array}$ & $\begin{array}{l}0.168 \\
(0.0892)\end{array}$ & $\begin{array}{l}0.186 \\
(0.139)\end{array}$ & $\begin{array}{l}0.597^{* * *} \\
(0.128)\end{array}$ & $\begin{array}{l}-0.149 \\
(0.106)\end{array}$ & $\begin{array}{l}0.0278 \\
(0.175)\end{array}$ \\
\hline Employee & $\begin{array}{l}-1.032^{* * *} \\
(0.0678)\end{array}$ & $\begin{array}{l}-0.964^{* * *} \\
(0.0809)\end{array}$ & $\begin{array}{l}-1.041^{* * *} \\
(0.125)\end{array}$ & $\begin{array}{l}-0.443^{* * *} \\
(0.111)\end{array}$ & $\begin{array}{l}-1.791^{* * *} \\
(0.104)\end{array}$ & $\begin{array}{l}-0.121 \\
(0.146)\end{array}$ \\
\hline Part-time job & $\begin{array}{l}-0.166^{* *} \\
(0.0535)\end{array}$ & $\begin{array}{l}0.109 \\
(0.111)\end{array}$ & $\begin{array}{l}-0.292^{* * *} \\
(0.0638)\end{array}$ & $\begin{array}{l}-0.278^{* * *} \\
(0.0647)\end{array}$ & $\begin{array}{l}-0.116 \\
(0.113)\end{array}$ & $\begin{array}{l}-0.169 \\
(0.186)\end{array}$ \\
\hline $\begin{array}{l}\text { Working } \\
\text { conditions }\end{array}$ & $\begin{array}{l}-0.0306 \\
(0.0394)\end{array}$ & $\begin{array}{l}-0.102^{*} \\
(0.0517)\end{array}$ & $\begin{array}{l}0.0638 \\
(0.0610)\end{array}$ & $\begin{array}{l}0.0932 \\
(0.0561)\end{array}$ & $\begin{array}{l}-0.0517 \\
(0.0710)\end{array}$ & $\begin{array}{l}-0.0581 \\
(0.0823)\end{array}$ \\
\hline Large city & $\begin{array}{l}0.00440 \\
(0.0410)\end{array}$ & $\begin{array}{l}-0.00452 \\
(0.0551)\end{array}$ & $\begin{array}{l}-0.0166 \\
(0.0615)\end{array}$ & $\begin{array}{l}0.0518 \\
(0.0600)\end{array}$ & $\begin{array}{l}0.0183 \\
(0.0774)\end{array}$ & $\begin{array}{l}-0.157 \\
(0.0848)\end{array}$ \\
\hline Medium city & $\begin{array}{l}-0.198^{* * *} \\
(0.0435)\end{array}$ & $\begin{array}{l}-0.309^{* * *} \\
(0.0574)\end{array}$ & $\begin{array}{l}-0.0697 \\
(0.0665)\end{array}$ & $\begin{array}{l}-0.371^{* * *} \\
(0.0650)\end{array}$ & $\begin{array}{l}-0.111 \\
(0.0798)\end{array}$ & $\begin{array}{l}-0.338^{* * *} \\
(0.0834)\end{array}$ \\
\hline Agriculture & $\begin{array}{l}0.281^{* * *} \\
(0.0824)\end{array}$ & $\begin{array}{l}0.277^{* *} \\
(0.0988)\end{array}$ & $\begin{array}{l}0.243 \\
(0.148)\end{array}$ & $\begin{array}{l}0.300^{*} \\
(0.144)\end{array}$ & $\begin{array}{l}0.960^{* * *} \\
(0.130)\end{array}$ & $\begin{array}{l}-0.626^{* * *} \\
(0.151)\end{array}$ \\
\hline Manufacturing & $\begin{array}{l}-0.0710 \\
(0.0445)\end{array}$ & $\begin{array}{l}-0.0218 \\
(0.0530)\end{array}$ & $\begin{array}{l}-0.244^{* *} \\
(0.0832)\end{array}$ & $\begin{array}{l}0.0156 \\
(0.0645)\end{array}$ & $\begin{array}{l}-0.185^{*} \\
(0.0808)\end{array}$ & $\begin{array}{l}-0.0818 \\
(0.0854)\end{array}$ \\
\hline $\begin{array}{l}\text { Personal } \\
\text { services }\end{array}$ & $\begin{array}{l}0.0882 \\
(0.0456)\end{array}$ & $\begin{array}{l}0.112 \\
(0.0756)\end{array}$ & $\begin{array}{l}0.0215 \\
(0.0586)\end{array}$ & $\begin{array}{l}0.340^{* * *} \\
(0.0610)\end{array}$ & $\begin{array}{l}-0.135 \\
(0.0839)\end{array}$ & $\begin{array}{l}-0.370^{* * *} \\
(0.108)\end{array}$ \\
\hline Blue collar & $\begin{array}{l}0.109^{*} \\
(0.0460)\end{array}$ & $\begin{array}{l}0.265^{* * *} \\
(0.0607)\end{array}$ & $\begin{array}{l}-0.0300 \\
(0.0746)\end{array}$ & $\begin{array}{l}-0.0113 \\
(0.0659)\end{array}$ & $\begin{array}{l}0.257^{* *} \\
(0.0830)\end{array}$ & $\begin{array}{l}0.0142 \\
(0.0915)\end{array}$ \\
\hline $\begin{array}{l}\text { Intellect. } \\
\text { worker }\end{array}$ & $\begin{array}{l}0.294^{* * *} \\
(0.0500)\end{array}$ & $\begin{array}{l}0.548^{* * *} \\
(0.0675)\end{array}$ & $\begin{array}{l}0.0297 \\
(0.0761)\end{array}$ & $\begin{array}{l}0.111 \\
(0.0654)\end{array}$ & $\begin{array}{l}0.387^{* * *} \\
(0.0938)\end{array}$ & $\begin{array}{l}0.242 \\
(0.129)\end{array}$ \\
\hline YEARSPNT & $\begin{array}{l}-0.0724^{* * *} \\
(0.00289)\end{array}$ & $\begin{array}{l}-0.0922^{* * *} \\
(0.00451)\end{array}$ & $\begin{array}{l}-0.0591^{* * *} \\
(0.00382)\end{array}$ & $\begin{array}{l}-0.0337^{* * *} \\
(0.00396)\end{array}$ & $\begin{array}{l}-0.132^{* * *} \\
(0.00477)\end{array}$ & $\begin{array}{l}-0.0461^{* * *} \\
(0.00983)\end{array}$ \\
\hline SRA & $\begin{array}{l}0.447^{* * *} \\
(0.00948)\end{array}$ & $\begin{array}{l}0.476^{* * *} \\
(0.0159)\end{array}$ & $\begin{array}{l}0.411^{* * *} \\
(0.0123)\end{array}$ & $\begin{array}{l}0.635^{* * *} \\
(0.0225)\end{array}$ & $\begin{array}{l}0.350^{* * *} \\
(0.0154)\end{array}$ & $\begin{array}{l}0.386^{* * *} \\
(0.0234)\end{array}$ \\
\hline $\begin{array}{l}\text { Pension } \\
\text { wealth }\end{array}$ & $\begin{array}{l}-0.00122^{* * *} \\
(0.0000551)\end{array}$ & $\begin{array}{l}-0.00146^{* * *} \\
(0.0000733)\end{array}$ & $\begin{array}{l}-0.000809^{* * *} \\
(0.0000859)\end{array}$ & $\begin{array}{l}-0.00115^{* * *} \\
(0.0000754)\end{array}$ & $\begin{array}{l}-0.00801^{* * *} \\
(0.000359)\end{array}$ & $\begin{array}{l}-0.0105^{* * *} \\
(0.00267)\end{array}$ \\
\hline constant & $\begin{array}{l}-1.709^{* *} \\
(0.617)\end{array}$ & $\begin{array}{l}-3.253^{* *} \\
(1.048)\end{array}$ & $\begin{array}{l}-0.0180 \\
(0.761)\end{array}$ & $\begin{array}{l}-15.62^{* * *} \\
(1.449)\end{array}$ & $\begin{array}{l}9.511^{* * *} \\
(1.091)\end{array}$ & $\begin{array}{l}1.927 \\
(1.617)\end{array}$ \\
\hline N & 44,558 & 24,493 & 20,065 & 18,905 & 16,719 & 8,934 \\
\hline$R^{2}$ & 0.115 & 0.105 & 0.097 & 0.083 & 0.169 & 0.179 \\
\hline
\end{tabular}

Notes: Standard errors in parentheses. ${ }^{*} p<0.05,{ }^{* *} p<0.01,{ }^{* * *} p<0.001$.

Model estimations also include the variable YEARSPNT (years spent working for pay or profit during working life). As pension systems in several countries include contribution years into calculations for pension entitlements, this variable could also be seen as related to pension systems. In all models, the estimated coefficient is highly significant and positive. The size of the effect is larger for men than for women and larger for poorer EU15 states than for richer or new EU states. 
Being an employee is associated with plans for an earlier retirement than observed for people working in their own business. This result is rather robust across model specifications and samples and corroborates earlier findings. Also working in a larger firm (which the LFS defines as 50 or more workers) is associated with plans for an earlier retirement, again robust across model specifications and samples and in line with results in the literature.

Results for part-time work and straining working conditions are mixed. The estimated coefficient for part-time work, as a rule, carries a negative sign. The significance of this effect seems to be driven by women and more wealthy countries, as estimations with more selected samples show (M2-M6). Some consideration was given to the fact that the sample also includes some people working only a very small number of hours per week. We therefore ran the models with a sample restricted to people regularly working at least 10 hours per week. Estimated results (not reported here) did not deviate considerably from results reported above. Working conditions turn out to be significant in very few models only. If significant, the coefficient is negative and the effect seems to be driven by male workers.

We now turn to the effects of industry and occupation. Estimations use dummy variables for two occupational groups: blue collar workers and intellectual workers (ISCO 1, 2; other white collar workers are the base category). For both variables, we estimate a significantly positive sign in both M1 models. The size of the effect is larger for intellectual workers. Segregation by sex leads to significant effects in the male sample only. Segregation by countries shows that the effect originates almost entirely from the less wealthy part of the EU15; for both other country groups, we fail to estimate significant effects.

In models not distinguishing between country type, we find planned retirement age in agriculture to be significantly higher than in other services, which was defined as a base category with regard to industries. Significance of this effect is lower for women than for men. But estimations for groups of countries show large deviations across Europe: The estimated coefficient for richer EU15 countries is about as high as for the whole sample, far larger and also positive in the poorer EU15 countries, but large and negative in new EU member states. Our hypothesis is that earlier planned retirement in new member states might be related to different (physically harder) working conditions, compared to richer countries.

In manufacturing, female workers and workers in poorer EU15 countries plan to retire earlier than workers in other services. In personal services, workers in rich EU15 countries plan to retire later, and workers in new EU member states plan to retire earlier than individuals working in the base category in their country group.

We now discuss the effects of pension system variables. As stated above, we use two variables to grasp characteristics of national pensions systems, standard retirement age (SRA) and either replacement rate (RR) or pension wealth (PW). In Models M1 through M6, variables are significant and with the expected sign: higher RR and PW are associated with plans for earlier retirement, while a higher SRA is associated with plans for later retirement. Segregation of the sample by sex (M2, M3) shows that the relationship between pension variables and planned age for retirement are slightly more pronounced in the male sample.

Segregation of the sample by country type reveals differences in the size of the effects: In M4 (EU 15, rich), the estimated coefficient for SRA is larger than in estimations for 
both other country groups. The effect of PW, in contrast, is largest in the new EU member states and smallest in rich EU15 countries.

\subsubsection{Joint retirement decision of partners}

In order to identify possible correlations between the partner's retirement plans, we reestimate the same type of models as above but with the additional variable "partner's planned age for retirement". We therefore need to restrict the sample to individuals that are married (or living in the same household with an adult of the opposite sex) and whose partner's data set is sufficiently complete as well. Additionally, we estimate models containing country fixed effects instead of pension system characteristics, as country fixed effects might better catch other relevant national characteristics.

As we are interested in only the joint retirement effect at this stage, Table 4 provides an overview of the relevant coefficient estimates ${ }^{3}$. Throughout all model and sample specifications, we find a consistently positive and significant coefficient for partner's planned retirement age. Thus, if one's partner plans to retire at a higher age, this is significantly and positively related to one's own plans for late retirement. Our results thus are in line with the literature suggesting joint household decisions rather than individual decisions are made with respect to retirement timing.

We estimate a significant effect for the male as well as for the female sample. Coefficients in the female sample are somewhat higher across all countries as well as in the three sub-sets of countries. The gender gap (in the size of the coefficient) in new EU MS is about double the size of corresponding gender gap in EU15 MS.

Differences between country groups are rather small. The joint retirement effects seem to be largest for women in new EU MS or poor EU15 MS, and they are smallest for men in rich EU15 and new EU MS.

The effect of the partner's retirement plan is rather strong as its inclusion into the regression equation raises the adj. R2 considerably ${ }^{4}$.

\section{Discussion and conclusion}

Population aging implies considerable challenges for Europe. The future size of the European labor force is an important production factor for economic growth. More flexible working time arrangements, more opportunities to update skills and better health/safety at the workplace are often mentioned as factors contributing to a longer working life. In the AHM2006, the workers are asked about the influence of these factors on postponing retirement. Flexible working time arrangements are mentioned by $20.7 \%$, followed by better health/safety at the workplace (14.6\%) and measures to update skills (10.5\%). Table 5 shows responses differentiated by country type. Within each factor, we observe striking differences between country groups. Differences are most pronounced regarding health and safety at the workplace: while about $10 \%$ of workers in rich EU15 countries state that improvements could delay their retirement, the corresponding share in new EU MS is 25\%. Poor EU15 MS hold an intermediate position in this respect. This "ranking" of country groups holds in all sub-groups of workers with regard to health and safety at the workplace but not necessarily with regard to more flexible working hours and better training opportunities.

Of the remaining two factors, flexible working times seem to be more important than improved training opportunities in each of the three country groups. In rich EU15 MS 
Table 4 Coefficient for partner's planned retirement age in linear regression model estimations for planned retirement age in alternative sample and model specifications

\begin{tabular}{|c|c|c|c|c|c|}
\hline & \multirow{2}{*}{$\begin{array}{l}\text { Country fixed } \\
\text { effects All countries }\end{array}$} & \multicolumn{4}{|c|}{ Pension variables: SRA, replacement rate } \\
\hline & & All countries & EU15, rich & EU15, poor & new EU members \\
\hline \multirow[t]{3}{*}{ Men } & $\begin{array}{l}0.328^{* * *} \\
(0.0137)\end{array}$ & $\begin{array}{l}0.323^{* * *} \\
(0.0135)\end{array}$ & $\begin{array}{l}0.300^{* * * *} \\
(0.0222)\end{array}$ & $\begin{array}{l}0.347^{* * *} \\
(0.0217)\end{array}$ & $\begin{array}{l}0.310^{* * *} \\
(0.0298 .)\end{array}$ \\
\hline & $N: 5,405$ & $N: 5,133$ & $N: 1,913$ & $\mathrm{~N}: 1,962$ & $N: 1,258$ \\
\hline & $R^{2}: 0.28$ & $R^{2}: 0.24$ & $R^{2}: 0.17$ & $R^{2}: 0.37$ & $R^{2}: 0.18$ \\
\hline \multirow[t]{3}{*}{ Women } & $\begin{array}{l}0.387^{* * *} \\
(0.0128)\end{array}$ & $\begin{array}{l}0.412^{* * *} \\
(0.0128)\end{array}$ & $\begin{array}{l}0.346^{* * *} \\
(0.0232)\end{array}$ & $\begin{array}{l}0.410^{* * *} \\
(0.0176)\end{array}$ & $\begin{array}{l}0.437^{* * *} \\
(0.0287)\end{array}$ \\
\hline & $N: 9,159$ & $N: 8,652$ & $\mathrm{~N}: 3,103$ & $N: 3,418$ & $\mathrm{~N}: 2,131$ \\
\hline & $R^{2}: 0.28$ & $R^{2}: 0.23$ & $R^{2}: 0.18$ & $R^{2}: 0.26$ & $R^{2}: 0.25$ \\
\hline \multirow[t]{5}{*}{ All } & $\begin{array}{l}0.331^{* * *} \\
(0.0094)\end{array}$ & $\begin{array}{l}0.369^{* * *} \\
(0.0092)\end{array}$ & $\begin{array}{l}0.322^{* * *} \\
(0.0163)\end{array}$ & $\begin{array}{l}0.390^{* * *} \\
(0.0136)\end{array}$ & $\begin{array}{l}0.353^{* * *} \\
(0.0206)\end{array}$ \\
\hline & $N: 14,564$ & N: 13,785 & $N: 5,016$ & $N: 5,380$ & $N: 3,389$ \\
\hline & $R^{2}: 0.26$ & $R^{2}: 0.24$ & $R^{2}: 0.18$ & $R^{2}: 0.29$ & $R^{2}: 0.32$ \\
\hline & & \multicolumn{4}{|c|}{ Pension variables: SRA, pension wealth } \\
\hline & & All countries & EU15, rich & EU15, poor & new EU members \\
\hline \multirow[t]{3}{*}{ Men } & & $\begin{array}{l}0.325^{* * *} \\
(0.0135)\end{array}$ & $\begin{array}{l}0.306^{* * *} \\
(0.0221)\end{array}$ & $\begin{array}{l}0.341^{* * *} \\
(0.0219)\end{array}$ & $\begin{array}{l}0.276^{* * *} \\
(0.0298)\end{array}$ \\
\hline & & $N: 5,133$ & $\mathrm{~N}: 1,913$ & $\mathrm{~N}: 1,962$ & $\mathrm{~N}: 1,258$ \\
\hline & & $R^{2}: 0.25$ & $R^{2}: 0.17$ & $R^{2}: 0.38$ & $R^{2}: 0.18$ \\
\hline \multirow[t]{3}{*}{ Women } & & $\begin{array}{l}0.412^{* * *} \\
(0.0129)\end{array}$ & $\begin{array}{l}0.347^{* * *} \\
(0.0233)\end{array}$ & $\begin{array}{l}0.392^{* * *} \\
(0.0180)\end{array}$ & $\begin{array}{l}0.437^{* * *} \\
(0.0287)\end{array}$ \\
\hline & & $N: 8,652$ & $\mathrm{~N}: 3,103$ & $\mathrm{~N}: 3,418$ & $\mathrm{~N}: 2,131$ \\
\hline & & $R^{2}: 0.23$ & $R^{2}: 0.17$ & $R^{2}: 0.27$ & $R^{2}: 0.25$ \\
\hline \multirow[t]{3}{*}{ All } & & $\begin{array}{l}0.369^{* * *} \\
(0.0092)\end{array}$ & $\begin{array}{l}0.323^{* * *} \\
(0.0163)\end{array}$ & $\begin{array}{l}0.376^{* * *} \\
(0.0138)\end{array}$ & $\begin{array}{l}0.365^{* * *} \\
(0.0205)\end{array}$ \\
\hline & & $N: 13,785$ & $\mathrm{~N}: 5,016$ & $N: 5,380$ & $N: 3,389$ \\
\hline & & $R^{2}: 0.24$ & $R^{2}: 0.18$ & $R^{2}: 0.30$ & $R^{2}: 0.33$ \\
\hline
\end{tabular}

Notes: Standard errors in parentheses. ${ }^{*} p<0.05,{ }^{* *} p<0.01,{ }^{* * *} p<0.001$. Control variables: as in Table 2 and Table 3.

and in new EU MS, flexible working times are mentioned more than twice as often as improved training opportunities, no matter whether the respondent works "normal" hours or shift, nights or weekend work.

All three factors, flexible working time, more training opportunities and better health/safety at the workplace, are more often felt to contribute to working longer among workers who wish to change their working hours, at least within EU15 states. In new EU MS, this difference is negligible.

Moreover, individuals who still work were asked for their reasons whey they stay at work. Again, we see remarkable differences between country groups (see Table 6). Less than $10 \%$ of workers in new EU MS, but $40 \%$ of workers in rich EU15 MS, state that they work for other than financial reasons, while more than $60 \%$ of workers in new EU MS and about $40 \%$ of workers in rich EU15 MS state that they work to generate sufficient household income.

It has been shown in the literature that characteristics of national pension systems like replacement rates and pension wealth both are correlated to retirement age (e.g., Gruber and Wise 1998; Blöndal and Scarpetta 1999; Blundell et al. 2002). Our paper is in line with these earlier findings but broadens the body of evidence in two ways. First, 
Table 5 Factors that would contribute to staying longer at work

\begin{tabular}{|c|c|c|c|c|c|c|c|c|c|}
\hline & \multicolumn{3}{|c|}{$\begin{array}{l}\text { More flexible } \\
\text { working time }\end{array}$} & \multicolumn{3}{|c|}{$\begin{array}{l}\text { More opportunities } \\
\text { to update skills }\end{array}$} & \multicolumn{3}{|c|}{$\begin{array}{l}\text { Better health/safety } \\
\text { at workplace }\end{array}$} \\
\hline & $\begin{array}{l}\text { EU15, } \\
\text { rich }\end{array}$ & $\begin{array}{l}\text { EU15, } \\
\text { poor }\end{array}$ & $\begin{array}{c}\text { New } \\
\text { EU MS }\end{array}$ & $\begin{array}{l}\text { EU15, } \\
\text { rich }\end{array}$ & $\begin{array}{l}\text { EU15, } \\
\text { poor }\end{array}$ & $\begin{array}{c}\text { New } \\
\text { EU MS }\end{array}$ & $\begin{array}{l}\text { EU15, } \\
\text { rich }\end{array}$ & $\begin{array}{l}\text { EU15, } \\
\text { poor }\end{array}$ & $\begin{array}{c}\text { New } \\
\text { EU MS }\end{array}$ \\
\hline \multicolumn{10}{|l|}{ Sex } \\
\hline Men & 23.3 & 19.4 & 23.7 & 8.9 & 13.7 & 12.2 & 9.5 & 15.6 & 26.8 \\
\hline Women & 23.2 & 19.9 & 24.7 & 11.6 & 14.4 & 11.3 & 10.3 & 12.7 & 23.9 \\
\hline \multicolumn{10}{|l|}{ Occupational group } \\
\hline Intellectual workers & 27.6 & 21.6 & 29.2 & 10.8 & 15.2 & 16.1 & 8.6 & 11.8 & 26.3 \\
\hline Other white collar & 23.0 & 20.5 & 24.7 & 11.3 & 15.6 & 11.9 & 10.0 & 13.2 & 23.1 \\
\hline Blue collar & 18.9 & 17.4 & 22.1 & 7.9 & 11.8 & 10.0 & 10.9 & 17.1 & 26.4 \\
\hline \multicolumn{10}{|l|}{ Industry } \\
\hline Agriculture & 14.2 & 12.0 & 18.1 & 5.8 & 7.8 & 9.8 & 9.0 & 14.9 & 21.4 \\
\hline Manufacturing & 20.9 & 18.9 & 23.6 & 7.8 & 12.3 & 10.9 & 9.5 & 16.4 & 27.3 \\
\hline Personal services & 26.9 & 21.7 & 26.2 & 13.8 & 16.7 & 13.5 & 12.4 & 14.3 & 24.9 \\
\hline Other services & 22.8 & 20.0 & 26.1 & 9.6 & 14.4 & 12.5 & 8.7 & 13.2 & 25.2 \\
\hline \multicolumn{10}{|l|}{$\begin{array}{l}\text { Wish to change } \\
\text { working hours }\end{array}$} \\
\hline no & 22.4 & 18.8 & 24.1 & 9.4 & 13.2 & 11.4 & 9.2 & 13.4 & 24.8 \\
\hline yes & 31.5 & 25.2 & 24.3 & 16.0 & 19.0 & 11.6 & 14.2 & 20.1 & 26.2 \\
\hline \multicolumn{10}{|l|}{ Working conditions } \\
\hline $\begin{array}{l}\text { No shift, night or } \\
\text { weekend work }\end{array}$ & 22.8 & 19.6 & 24.1 & 9.9 & 14.1 & 11.9 & 9.2 & 13.7 & 25.0 \\
\hline $\begin{array}{l}\text { Shift, night or } \\
\text { weekend work }\end{array}$ & 24.7 & 19.5 & 24.7 & 11.0 & 13.4 & 11.8 & 12.0 & 17.3 & 26.5 \\
\hline $\begin{array}{l}\text { Average hours } \\
\text { worked }\end{array}$ & 36.2 & 39.8 & 40.9 & 35.6 & 38.4 & 40.6 & 35.9 & 39.4 & 40.8 \\
\hline Total - \% & 23.3 & 19.6 & 24.2 & 10.2 & 14.0 & 11.8 & 9.9 & 14.4 & 25.4 \\
\hline Total - N & 21,817 & 25,103 & 20,175 & 21,965 & 25,308 & 20,185 & 22,000 & 25,369 & 20,188 \\
\hline
\end{tabular}

Note: Percentages of people in the study group who answered "yes", i.e., they assume that the respective factor would contribute to a person staying longer at work. Source: AHM 2006.

we provide evidence for planned rather than actual retirement age. If pension systems of the future are to be designed in a way that workers intend to work longer rather than are more or less forced to do so by regulations, this approach of using planned retirement may provide useful insights. Our analyses, for instance, confirm that in new EU MS, planning to retire later is not only a financial issue, but more than in other EU countries, it is also a question of health and safety at the workplace. Investments in this area, therefore, presumably will have a favorable side-effect apart from lower suffering, i.e., later retirement. Second, our analysis is more comprehensive than many studies (e.g., Machado and Portela 2014 focusing on Portugal; Blekesaune and Solem 2005 focusing on Norway) as it is not limited to a single country but uses data containing a broad set of European countries and allows for comparisons between country groups.

Our estimations for separate groups of countries underline the interrelation of individual retirement plans with the general environment ${ }^{5}$. In richer countries of the EU15, standard retirement age seems to have a larger effect on planned retirement age than in poorer EU15 states or in new EU member states. The effect of pension wealth, in contrast, is largest in the new EU member states and smallest in rich EU15 countries. 
Table 6 Main financial incentive to stay at work

\begin{tabular}{lccc}
\hline & EU15, rich & EU15, poor & New EU MS \\
\hline Increase retirement pension entitlement & 14.9 & 22.3 & 16.9 \\
Men & 14.2 & 25.7 & 36.1 \\
Women & 14.6 & 23.5 & 30.7 \\
Total & & & \\
Provide sufficient household income & 41.4 & 59.6 & 68.5 \\
Men & 38.2 & 58.0 & 58.3 \\
Women & 40.0 & 59.0 & 61.2 \\
Total & & & \\
No financial incentive & 43.7 & 18.1 & 14.6 \\
Men & 47.6 & 16.3 & 5.6 \\
Women & 45.5 & 17.5 & 8.2 \\
Total & & & \\
\hline
\end{tabular}

Note: Percentages of people in the study group who indicated the respective incentive which made them stay at work. Source: Author's calculation using AHM 2006.

The relative generosity of the pension system, therefore, seems to be of higher importance for individual retirement decisions in less wealthy countries ${ }^{6}$.

Choice of variable (pension wealth or replacement rate) hardly affects results. This is somewhat unexpected as pension wealth typically is seen as the stronger variable, but it may be explained by the regrettable fact that we can include these variables only in the form of country averages - albeit differentiated by sex - rather than as individually calculated information.

Our analyses take, inter alia, information on two occupational groups into consideration: blue collar workers and intellectual workers. We find for members of both groups that they plan to retire later than other workers, with the effect for intellectual workers being stronger than that for blue collar workers. We would assume that reasons for late retirement are different in both groups of workers: In some countries (with stronger insurance and contribution elements in the pension system), intellectual workers' typically later entry into full-time employment may contribute to later entitlements for pension. But during their career, intellectual workers often earn higher wages and feel less physical strain from work, which both may contribute to retirement needs occurring at older ages compared to other workers.

Blue collar workers, on the other hand, may work longer due to lower wages during their active time, which makes accumulation of savings for retirement harder. This explanation could be more relevant in poorer EU15 states compared to richer EU15 states. In richer states, pension systems assumedly enable more retirees to live from pensions alone, or wage levels facilitate the accumulation of sufficient savings to retire earlier. Our data set provides some backing for this interpretation: less than $10 \%$ of workers in new EU MS, but 40\% of workers in rich EU15 MS, state that they work for other than financial reasons, while more than $60 \%$ of workers in new EU MS and about $40 \%$ of workers in rich EU15 MS state that they work to generate sufficient household income.

Estimating segregated models for groups of countries shows that type of work matters most in the less wealthy part of the EU15; for both other country groups, we fail to estimate significant effects. Furthermore, these effects seem to be more relevant for 
men than for women, presumably because female careers more often also depend on a larger number of other, and not necessarily employment-related, factors.

We also find different industry effects in different types of countries. Workers in agriculture plan to retire earlier than workers in services if living in new EU states, but later if living in the EU15. This may be related to different working conditions in this sector in different parts of Europe. Our hypothesis is that earlier planned retirement in new member states might be triggered by different (physically harder) working conditions compared to richer countries. This is also corroborated by the observations that in new EU MS, better precautions regarding health more often could contribute to extending active careers than in other EU MS. Similarly, workers in social or personal services plan to retire relatively late in rich EU15 states but earlier in new EU states.

If current transitions in labor markets also include moving from physically demanding to intellectually demanding work environments (Fischer-Kowalski et al. 2012), this could contribute to workers remaining active in the labor market longer. This would not necessarily require a change in the structure of the economy but rather would require the implementation of improvements in existing sectors. This interpretation, however, deserves further research using databases which can identify strenuous work conditions more clearly.

Another often-mentioned transition in labor markets is the increasing share of shortterm and part-time jobs. Working part-time was recommended as a means towards easier reconciling strenuous work and limited health or strength of older workers (e.g., European Commission 2012). In line with existing evidence regarding part-time work and (early) retirement (Machado and Portela 2014; Graf et al. 2011), our results also do not support this view. Quite contrary, we find a negative association between working part-time and planned retirement age. Nikolova and Graham (2014) point out that analyzing the number of hours worked might only blur the picture, as it has been shown that voluntary part-time work of older workers actually has the potential to increase individual well-being over and above that of comparable retirees. Involuntary part-time workers were not found to experience such a "well-being premium". Similarly, we would assume that the nature of part-time work - voluntary or involuntary - is of importance when trying to use part-time options as an incentive to work longer.

In line with the existing literature, we find a significant relation between two partners' planned retirement age. While other authors mostly concentrated on actual retirements, which may be blurred by pension entitlements or job requirements, our results indicate some degree of matching between partners already at the stage of retirement planning. In contrast to some studies of the joint effect in actual retirement, we find a significant effect not only for women but also for men, and for both, of comparable effect size. As Hospido and Zamarro (2014) point out in the context of actual joint retirement decisions of couples, failing to take the joint retirement effect into account may lead to overestimations of the effect policy can achieve by raising the statutory retirement age.

The joint retirement effects were found to stem predominantly from countries where gender differences in labor market participation are small (Hospido and Zamarro 2014). Hence, we find concerns regarding overestimations in particular for countries whose policy to raise statutory retirement age rests mostly or entirely on adjusting the female retirement age to the male one. However, we have to admit that the lack of the exact age of observed individuals limits the accuracy of our analysis as we are not able to identify for which calendar year a person's and the partner's retirement are planned. Furthermore, we 
need to stress that we do not investigate causal relationships. That is, we cannot distinguish if a partner's plan to retire at an older age contributes to postponing the other partner's retirement age or if the estimation results simply reflect a matching effect of partners with similar preferences regarding leisure and work.

We did some sensitivity tests to infer the stability of our main results. We extended our sample to all age groups, we included country fixed effects and we used duration analysis techniques (cox model). In general, our results are robust with respect to the various determinants. The results are available on request.

Overall, our results are in line with the literature with respect to measures to postpone retirement. Financial incentives are important, quality of the work and joint retirement effects also play a role for planned retirement age. Looking at various countries within Europe shows that the interactions between planned retirement age and personal and workrelated variables vary across Europe. To achieve the necessary extension of the working life, policy measures have to take these differences into account.

\section{Endnotes}

${ }^{1}$ Participation was compulsory in a very heterogeneous group of countries, consisting of Belgium, Spain, France, Italy, Cyprus, Malta, Portugal, and Slovakia. Generally, response rates were also very high in the remaining countries, with a unit non-response rate of 13\% in Austria, by far the highest non-response rate (Eurostat 2008). Owing to high response rates, we do not expect that differences in response patterns lead to biased cross-country comparisons.

${ }^{2} 44 \%$ of all observations have been recoded. We perform robustness checks by using extreme values (55, 60, 65 and 59,64, 69, respectively) and find quantitatively unchanged results.

${ }^{3}$ The referee suggested that the influence of the partner's planned retirement age could be at least partly spurious if one partner had been interviewed in place of the other. We conduct some robustness checks (a regression controlling for proxy interviews, excluding proxy interviews from the sample) to test for that hypothesis. Overall, we find that the estimated influence of the partner's planned retirement age is very robust with respect to proxy interviews.

${ }^{4}$ E.g., the $R\left({ }^{2}\right)$ for the regression with country fixed effects drops from 0.26 to 0.17 when the partner's planned retirement age is excluded.

${ }^{5}$ We tested for statistical significance of differences between country groups by running a regression with the full sample where all variables were segregated by country group (interactions of original variables and dummies representing two country groups).

${ }^{6}$ Note that we have only country level information on pension wealth. 


\section{Author details}

${ }^{1}$ Institute for Advanced Studies, Stumpergasse 56, 1060 Vienna, Austria. ${ }^{2}$ Vienna University of Economics and Business, Welthandelsplatz 1, 1020 Vienna, Austria.

Received: 4 April 2014 Accepted: 10 November 2014

Published online: 12 February 2015

\section{References}

Adams GA, Prescher J, Beehr TA, Lepisto L (2002) Applying work-role attachment theory to retirement decision-making Int J Aging Hum Dev 54(2):125-137

Alavinia SM, Burdorf A (2008) Unemployment and retirement and ill-health: a cross sectional analysis across European Countries. Int Arch Occup Environ Health 82(1):39-45

Almenberg J, Säve-Söderbergh J (2011) Financial literacy and retirement planning in Sweden. J Pension Econ Finance 10(4):585-598

Beehr TA, Glazer S, Nielson NL, Farmer SJ (2000) Work and nonwork predictors of employees' retirement ages. J Vocat Behav 57:206-225

Benitez-Silva H, Dwyer DS (2005) The rationality of retirement expectations and the role of new information. Rev Econ Stat 87(3):587-592

Bernheim BD (1987) The Timing of Retirement: A Comparison of Expectations and Realizations. In: Wise DA (ed) The Economics of Aging. University of Chicago Press, Chicago, pp 335-358

Blekesaune M, Solem PE (2005) Working conditions and early retirement: a prospective study of retirement behavior. Res Aging 27(1):3-30

Blöndal S, Scarpetta S (1999) The Retirement Decision in OECD Countries. OECD Economics Department Working Papers No. 202., doi:10.1787/565174210530

Blundell R, Meghir C, Smith S (2002) Pension incentives and the pattern of early retirement. Econ J 112:153-170

Bottazzi R, Jappelli T, Padula M (2006) Retirement expectations, pension reforms, and their impact on private wealth accumulation. J Public Econ 90(12):2187-2212

Brown JR (2009) Understanding the Role of Annuities in Retirement Planning. In: Lusardi A (ed) Overcoming the Saving Slump: how to Increase the Effectiveness of Financial Education and Saving Programs. University of Chicago Press, Chicago, pp 178-206

Bucher-Koenen T, Lusardi A (2011) Financial literacy and retirement planning in Germany. J Pension Econ Finance 10 (4):565-584

Bütler M, Huguenin O, Teppa F (2004) What Triggers Early Retirement? Results from Swiss Pension Funds. CERP Working Paper No. 35/04., http://web.econ.unito.it/cerp/Pubblicazioni/archivio/WP_CeRP/WP_35.pdf. Accessed 31 Oct 2014

Clark RL, Morrill MS, Allen SG (2012) The role of financial literacy in determining retirement plans. Econ Inq 50(4):851-866

Coppola M, Wilke CB (2014) At what age do you expect to retire? retirement expectations and increases in the statutory retirement Age. Fisc Stud 35(2):165-188

de Grip A, Fouarge D, Montizaan R (2013) How sensitive are individual retirement expectations to raising the retirement age? De Economist 161(3):225-251

Disney R, Tanner S (1999) What can we Learn from Retirement Expectations Data? The Institute for Fiscal Studies Working Paper Series No. W99/17, doi:10.1920/wp.ifs.1999.9917

Dorn D, Sousa-Poza A (2005) The determinants of early retirement in Switzerland. Swiss J Econ Stat 141(2):247-283

Dorn D, Sousa-Poza A (2010) Voluntary and involuntary early retirement: an international analysis. Appl Econ 42(4):427-438

Dwyer DS, Mitchell OS (1999) Health problems as determinants of retirement: Are self-rated measures endogenous? Health Econ 18(2):173-193

Elovainio M, Forma P, Kivimäki M, Sinervo T, Sutinen R, Laine M (2005) Job demands and job control as correlates of early retirement thoughts in Finnish social and health care employees. Work stress. Int J Work Health Org 19(1):84-92

European Commission (2011) Demography Report 2010 - Older, More Numerous and Diverse Europeans. Commission Staff Working Document, Brussels, http://epp.eurostat.ec.europa.eu/portal/page/portal/population/documents/Tab/ report.pdf. Accessed 4 Nov 2014

European Commission (2012) An Agenda for Adequate, Safe and Sustainable Pensions - White Paper. Commission Staff Working Document, Brussels, http://eur-lex.europa.eu/LexUriServ/LexUriServ.do?uri=COM:2012:0055:FIN:EN:PDF. Accessed 4 Nov 2014

Eurostat (2008) Transition from Work into Retirement. Eurostat Methodologies and Working Papers, Luxembourg, http://epp.eurostat.ec.europa.eu/portal/page/portal/employment_unemployment_Ifs/documents/Evaluation\% 20report\%20AHM\%202006.pdf. Accessed 31 Oct 2014

Fischer JAV, Sousa-Poza A (2006) The Institutional Determinants of Early Retirement in Europe, University of St. Gallen Department of Economics Discussion Paper No. 2006-08., http://www.researchgate.net/publication/ 23696272_The_Institutional_Determinants_of_Early_Retirement_in_Europe/file/d912f50ad435c45339.pdf. Accessed 31 Oct 2014

Fischer-Kowalski M, Haas W, Wiedenhofer D, Weisz U, Pallua I, Possanner N, Behrens A, Serio G, Alessi M, Weis E (2012) Socio-Ecological Transitions: Definition, Dynamics and Related Global Scenarios. Key Assumptions on SET, Neujobs State of the Art Report No.6/D1.1., http://www.neujobs.eu/sites/default/files/publication/2012/05/wp1_Socio-ecological\%20transitions\%20and\%20global\%20scenarios.pdf. Accessed 4 Nov 2014

Graf N, Hofer H, Winter-Ebmer R (2011) Labour supply effects of a subsidised Old-Age part-time scheme in Austria. J Labour Market Res 44(3):217-229

Gruber J, Wise D (1998) Social security and retirement. An international comparison. Am Econ Rev 88(2):158-163

Gustman A, Steinmeier T (2000) Retirement in dual-career families: a structural model. J Labor Econ 18(3):503-545 
Hospido L, Zamarro G (2014) Retirement patterns of couples in Europe. IZA J Eur Labor Stud 3:12, doi:10.1186/21939012-3-12

Hurd MJ (1990) The Joint Retirement Decision of Husbands and Wives. In: Wise DA (ed) Issues in the Economics on Aging. University of Chicago Press, Chicago, pp 231-258

Jones AM, Rice N, Roberts J (2010) Sick of work or too sick to work? Evidence on self-reported health shocks and early retirement from the BHPS. Econ Modelling 27(4):866-880

Karpansalo M, Manninen P, Kauhanen J, Lakka TA, Salonen JT (2004) Perceived health as a predictor of early retirement. Scand J Work Environ Health 30(4):287-292

Lusardi A, Mitchell OS (2011) Financial Literacy and Planning: Implications for Retirement Wellbeing. In: Lusardi A, Mitchell OS (eds) Financial Literacy. Implications for Retirement Security and the Financial Marketplace. Oxford University Press, New York, pp 17-39

Machado CS, Portela M (2014) Hours of work and retirement behavior. IZA J Eur Labor Stud 3:16, doi:10.1186/2193-9012-3-16

McGarry K (2004) Health and retirement - Do changes in health affect retirement expectations? J Hum Resour 39 (3):624-648

Moen P, Sweet S, Swisher R (2005) Embedded career clocks: the case of retirement planning. Adv Life Course Res 9:237-265

Montalto CP, Yuh Y, Hanna S (2000) Determinants of planned retirement age. Fin Serv Rev 9(1):1-15

Nikolova M, Graham C (2014) Employment, late-life work, retirement, and well-being in Europe and the United States. IZA J Eur Labor Stud 3:5, doi:10.1186/2193-9012-3-5

OECD (2011) Pensions at a Glance. Retirement-Income Systems in OECD and G20 Countries. OECD Publishing, doi:10.1787/19991363

Parnes HS, Sommers DG (1994) Shunning retirement: work experience of men in their seventies and early eighties. J Gerontol 49(3):117-124

Pienta AM, Hayward MD (2002) Who expects to continue working after 62? The retirement plans of couples. J Geronto B Psychol Sci Soc 57(4):199-208

Schnalzenberger M, Schneeweis N, Winter-Ebmer R, Zweimüller M (2008) Job Quality and Retirement Decisions. In: Börsch-Supan A, Brugiavini A, Jürges H, Kapteyn A, Mackenbach J, Siegrist J, Weber G (eds) First results from the Survey of Health, Ageing and Retirement in Europe (2004-2007). Mannheim Research Institute for the Economics of Aging, Mannheim, pp 215-221

Siegrist J, Wahrendorf M, von dem Knesebeck O, Jürges H, Börsch-Supan A (2007) Quality of work, well-being, and intended early retirement of older employees—baseline results from the SHARE Study. Eur J Public Health 17(1):62-68

van Dam K, van der Vorst JD, van der Heijden BI (2009) Employees' intentions to retire early - a case of planned behavior and anticipated work conditions. J Career Dev 35(3):265-289

van den Berg T, Elders LA, Burdorf A (2010) Influence of health and work on early retirement. J Occup Environ Med 52(6):576-583

van Rooij MC, Lusardi A, Alessie RJM (2011) Financial literacy and retirement planning in the Netherlands. J Econ Psychol 32(4):593-608

van Solinge H, Henkens K (2009) Living longer, working longer? The impact of subjective life expectancy on retirement intentions and behaviour. Eur J Public Health 20(1):47-51

Vonkova H, van Soest A (2014) How sensitive are retirement decisions to financial incentives: a stated preference analysis. J Appl Econometr 29(2):246-264

Zappalà S, Depolo M, Fraccaroli F, Guglielmi D, Sarchielli G (2008) Postponing job retirement?: Psychosocial influences on the preference for early or late retirement. Career Dev Int 13(2):150-167

Zweimüller J, Winter-Ebmer R, Falkinger J (1996) Retirement of spouses and social security reform. Eur Econ Rev 40(2):449-472

\section{Submit your manuscript to a SpringerOpen ${ }^{\circ}$ journal and benefit from:}

- Convenient online submission

- Rigorous peer review

- Immediate publication on acceptance

- Open access: articles freely available online

- High visibility within the field

- Retaining the copyright to your article 